\title{
Una introducción al enfoque del curso de vida y su uso en la investigación pediátrica: principales conceptos y principios metodológicos
}

\author{
Francisco J. Cenobio-Narcizo*, Jessica H. Guadarrama-Orozco, Gerónimo Medrano-Loera, \\ Karina Mendoza-de la Vega y Daniela González-Morales \\ Departamento de Cuidados Paliativos y Calidad de Vida, Hospital Infantil de México Federico Gómez, Ciudad de México, México
}

\begin{abstract}
Resumen
Desde su concepción, el enfoque del curso de vida se ha utilizado ampliamente en la investigación sociodemográfica y en los estudios sociológicos. El empleo de esta perspectiva se ha difundido desde hace algunos años en la investigación en salud. Sin embargo, su uso ha sido muy limitado en pediatría. Este trabajo presenta una exposición de los principales conceptos del enfoque del curso de vida y algunos principios metodológicos para el desarrollo de investigaciones desde esta perspectiva. Además, se incluyen una serie de proyectos de investigación que se han valido de este enfoque como fundamentación conceptual en su diseño e implementación y algunas fuentes de información que pueden ser utilizadas para el desarrollo de investigaciones desde esta conceptualización en México.
\end{abstract}

Palabras clave: Enfoque del curso de vida. Trayectoria. Transición. Longitudinal y punto de inflexión.

\section{An introduction to the life course approach and its use in pediatric research: main concepts and methodological principles}

\begin{abstract}
Since its inception, the life course approach has been widely used in the socio-demographic research and sociological studies. The use of this perspective in health research has spread for some years, although its use in pediatrics has been limited. This work presents the main concepts of the life course approach and some methodological principles for the development of research from this perspective. In addition, a series of research projects that have used this approach as a conceptual basis in their design and implementation are included, as well as some sources of information that can be used for the development of research since this conceptualization in Mexico.
\end{abstract}

Key words: Life course approach. Trajectory. Transition. Longitudinal and turning point.

Correspondencia:

*Francisco Javier Cenobio-Narcizo

E-mail: neofjcn01 @gmail.com
Disponible en internet: 19-07-2019 Bol Med Hosp Infant Mex.2019;76:203-209 www.bmhim.com 


\section{Introducción}

El enfoque del curso de vida ha sido, desde su concepción, una perspectiva multidisciplinaria, pues se nutre de la demografía, sociología, historia, psicología, antropología, biología y epidemiología ${ }^{1-3}$ para su formulación conceptual. Este enfoque da cuenta de un complejo proceso de cambios en las trayectorias vitales de la población, derivados de eventos en el contexto social e individual de los sujetos, además de que entrelaza el tiempo histórico y biológico para analizar la manera en la que estos configuran la forma de pensar, sentir y actuar. Por lo mismo, es fundamental para estudiar las etapas de desarrollo, desde la infancia hasta la vejez $z^{4-6}$.

El enfoque del curso de vida se utiliza para entender los cambios en la vida de la población, considerando sucesos de larga duración; es decir, sucesos que acontecen a lo largo de la vida con un calendario y una intensidad determinada por el momento histórico que se vive y las condiciones propias de la población ${ }^{7-10}$. Esta perspectiva ha contribuido a la comprensión de cómo el pasado y presente configuran la vida de la población y cómo las distintas etapas vitales contribuyen a configurar el estado general de salud ${ }^{9-11}$. Los distintos eventos o cambios que experimenta una persona tienen efectos diversos dependiendo de su edad, aunque están en juego otros factores relevantes que esta conceptualización reconoce, como el sexo, la raza, la etnia y la clase social dentro de un tiempo y una sociedad determinados $5,12,13$.

Desde la publicación de los primeros trabajos en 1970, el enfoque del curso de vida ha recibido importantes críticas. Se minimizaba su capacidad analítica, pues se argumentaba que esta conceptualización era sentido común, por lo que su promoción era innecesaria $^{14}$; sumado a esto, el señalamiento de que la posición de este enfoque era determinística o fatalista para los individuos con situaciones adversas durante el desarrollo de su vida ${ }^{12}$. A pesar de estas críticas, el enfoque del curso de vida ha ganado un lugar importante en la academia, entre los expertos en salud pública y los tomadores de decisiones ${ }^{13,14}$.

El objetivo de este trabajo fue realizar una exposición de los aspectos conceptuales y metodológicos del enfoque del curso de vida y cómo estos pueden utilizarse en la investigación en salud y, en particular, en la investigación pediátrica. Se presentan también algunos trabajos que se han desarrollado desde esta perspectiva, las consideraciones metodológicas y una selección de fuentes de información que se pueden utilizar en México para el desarrollo del enfoque del curso de vida en la práctica.

\section{Características del enfoque y principales postulados}

El enfoque del curso de vida tiene distintos postulados y principios que conforman su construcción conceptual. Algunos de ellos han cambiado o se han nutrido del avance de distintas disciplinas y metodologías novedosas, pero, en esencia, han permanecido desde los inicios de su concepción. En la actualidad existe una controversia con respecto al adecuado uso conceptual del enfoque del curso de vida, ya que este término es utilizado de forma indistinta por diferentes investigadores $^{15}$. En este trabajo se utiliza la conceptualización desarrollada por G. Elder, con sus posteriores modificaciones y adiciones conceptuales ${ }^{4,6}$.

Los postulados que conforman el enfoque del curso de vida son los siguientes: transición, trayectoria y punto de inflexión (turning point). Sumados estos postulados se encuentran cinco principios: desarrollo a largo plazo, principio de tiempo y lugar, principio de tiempo (timing), principio de vidas interconectadas y el libre albedrío (agency). Algunos trabajos incorporan conceptos adicionales o reagrupan los principios de este enfoque, pero el cuerpo analítico está formado fundamentalmente de estos elementos ${ }^{4-6,16}$.

El concepto de transición hace referencia a cambios de estado, posición o situación, no necesariamente predeterminados 0 absolutamente previsibles, aunque algunos de estos tienen mayores o menores probabilidades de ocurrir por el cambio en la edad y las condiciones culturales o sociales de cada individuo ${ }^{16}$. Las transiciones representan un cambio del rol y el estatus que se tenía con anterioridad, para iniciar otro con nuevos derechos y obligaciones; a veces puede implicar nuevas facetas de identidad social ${ }^{5,16}$. Durante la vida, se experimentan una gran cantidad de transiciones. Por ejemplo, el inicio de un nuevo grado académico, el primer empleo, el inicio de la primera unión o el nacimiento del primer hijo.

La trayectoria hace referencia a un camino de larga duración en el tiempo, que no necesariamente es permanente y estable, pero que se espera que tenga cierta continuidad. Para el enfoque del curso de vida, la trayectoria no supone alguna secuencia en particular ni determinada velocidad en el proceso del propio tránsito, aunque sí existen mayores o menores probabilidades en el desarrollo de ciertas trayectorias vitales. La trayectoria puede verse como un proceso: de la 
infancia a la vejez. En ella, pueden acontecer múltiples transiciones; por ello se dice que las transiciones siempre están integradas en las trayectorias ${ }^{4-6,16}$. Por ejemplo, ciertos eventos vitales pueden acarrear resultados de largo plazo: el contraer matrimonio es una transición que puede desatar una serie de cambios en la trayectoria vital de una persona, pues puede ser el inicio de la fecundidad, entrar al mercado laboral, fin de la etapa escolar, entre otros.

El punto de inflexión es un momento en el que se producen cambios importantes en la trayectoria del ciclo de vida 5 . Se puede afirmar que un punto de inflexión se trata de eventos que provocan fuertes modificaciones en la dirección del ciclo de una vida. Estos cambios pueden surgir de acontecimientos fácilmente identificables, o bien de situaciones que se pueden catalogar de subjetivas; en cualquier caso, se presenta un cambio que implica la discontinuidad en una o más de las trayectorias vitales ${ }^{16}$. El punto de inflexión sirve como un cambio duradero y no solamente como un desvío temporal, ya que ubica a los sujetos y a sus familias en otra perspectiva distinta a la que se tenía con anterioridad ${ }^{5}$.

Las transiciones no siempre producen un punto de inflexión. Sin embargo, una transición puede considerarse un punto de inflexión con el paso del tiempo. En comparación con las trayectorias o las transiciones, el punto de inflexión no puede ser previsto, ya que es un suceso no contemplado de forma anticipada por un sujeto: el reconocimiento se debe realizar de forma retrospectiva después de haber vivido el acontecimiento ${ }^{5,16}$. Un ejemplo del punto de inflexión puede ser el diagnóstico de una enfermedad grave, ya que cambia al individuo en su cotidianeidad, en sus hábitos y la estructura de su familia.

A continuación, se enlistan los principios que integran el enfoque del curso de vida:

- Tiempo y lugar históricos. Los individuos están incrustados y son moldeados por los tiempos históricos y lugares en los que se encuentran a lo largo de su vida.

- El tiempo en las vidas. El impacto de una sucesión de transiciones o eventos depende de cuándo ocurren en la vida de una persona. Los roles y comportamientos están asociados con grupos de edad particulares, según la edad biológica, psicológica, social y espiritual.

- Vidas vinculadas o interconectadas. Las vidas se viven de manera interdependiente y las influencias sociales e históricas se expresan a través de esta red de relaciones compartidas. Las vidas humanas son interdependientes y la familia es el escenario principal para experimentar e interpretar fenómenos históricos, culturales y sociales más amplios.

- La agencia humana (human agency). Afirma que los individuos construyen su propia vida a través de las elecciones y acciones que toman dentro de las limitaciones y oportunidades de la historia que viven y las circunstancias sociales en las que se encuentran ${ }^{5,16}$. La información longitudinal ha sido uno de los aportes esenciales para el enfoque del curso de vida. Por su naturaleza, estas fuentes de información ayudan a realizar inferencias válidas sobre distintos fenómenos sociales y de salud que le acontecen a la población. La célebre frase que señala que «el pasado condiciona muchos de los eventos futuros de la población» dejó de ser una sentencia ligera, ya que ahora se puede demostrar su validez con evidencia cuantitativa y cualitativa sólida ${ }^{5,17}$. El dar seguimiento a los mismos individuos, conforme van creciendo y envejeciendo, proporciona información y puntos de vista diferentes a los estudios longitudinales ${ }^{16}$.

Los estudios longitudinales son el estándar de oro para el enfoque del curso de vida. Sin embargo, se deben aceptar una serie de limitaciones prácticas en el momento de llevarlos a cabo, ya que la mayoría de estos son extremadamente costosos. Por ello, se ha optado por considerar como información longitudinal aquella que puede provenir de encuestas, tanto de carácter prospectivo como retrospectivo ${ }^{16,17}$. Además de los obstáculos financieros que limitan los estudios longitudinales, se pueden señalar problemas de pérdida de contacto con la muestra o de no respuesta. Pese a estas dificultades, en EE.UU. y Europa se ha impulsado la publicación de diversos estudios siguiendo el enfoque del curso de vida con datos longitudinales ${ }^{18,19}$. Además, se han promovido publicaciones especializadas siguiendo esta perspectiva, como Longitudinal and Life Course Studies y Advances in Life Course Research.

\section{El enfoque del curso de vida y la investigación en salud}

En las últimas dos décadas se han desarrollado una serie de investigaciones que unen la perspectiva del

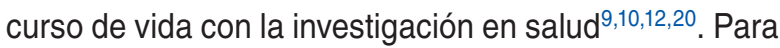
entender la salud, en el sentido más amplio, se debe entender la relación entre lo social, el aspecto psicológico y los factores biológicos. La comprensión de estos aspectos y su vínculo contribuyen al análisis de los factores que determinan la morbilidad y mortalidad de 
la población ${ }^{9}$. En este sentido, el enfoque del curso de vida vincula los aspectos sociales, psicológicos y biológicos, tanto en el ámbito académico como en el diseño e implementación de políticas públicas ${ }^{9,10,20,21}$.

Uno de los desarrollos más importantes que provienen del enfoque del curso de vida es el concepto de ventaja/desventaja acumulada (o también llamada divergencia o desigualdad acumulada). Este concepto analiza cómo las disparidades en la salud a lo largo de la vida pueden ser el resultado de las desigualdades de base socioeconómica, pues los individuos soportan los efectos acumulativos de los comportamientos de su vida y las experiencias económicas y psicosociales sobre su salud ${ }^{5,21-23}$. La idea de que la desventaja aumenta la exposición al riesgo y la ventaja aumenta la exposición a la oportunidad indica que un mayor estrato socioeconómico ayudaría a reducir la morbilidad y la incapacidad en los últimos años de vida, y que lo contrario sucede con un estrato socioeconómico más bajo, ya que aumentaría el riesgo de mayor discapacidad y peores condiciones de salud ${ }^{5,23}$.

Otro de los estudios importantes que se han desarrollado para relacionar el enfoque del curso de vida con la investigación en salud es el correspondiente a la hipótesis de los orígenes fetales o programación. Esta hipótesis intenta vincular las condiciones en el entorno intrauterino con el desarrollo posterior de enfermedades crónicas en adultos ${ }^{1,11,12,22}$. La hipótesis de programación se originó en la explicación de cómo determinados sucesos sociales traumáticos o que llevaron a la población a vivir en situaciones extremas pudieron afectar a generaciones posteriores; es decir, se pueden expresar cambios en la epigenética de una determinada población. Las distintas guerras, hambrunas y sufrimiento humano del siglo pasado pudieron influenciar la programación de la salud y las enfermedades durante el periodo fetal y la primera infancia. Por ejemplo, se considera el caso de las tendencias epidemiológicas de las enfermedades cardiovasculares y los factores de riesgo en Rusia y Europa del Este 1,12,21,24. Además, se ha postulado, con evidencia reciente, la existencia de periodos críticos o sensibles de crecimiento y desarrollo, no solamente en el útero y la primera infancia, sino también durante la infancia y la adolescencia, cuando las exposiciones ambientales causan más daño a la salud y pueden tener efectos a largo plazo. Es en estos periodos críticos cuando las habilidades, hábitos, estrategias de afrontamiento, actitudes y valores sociales se adquieren más fácilmente (comparado con edades posteriores). Estas habilidades influyen fuertemente en las trayectorias del curso de la vida, con implicaciones importantes para la salud en la vida posterior ${ }^{9,11,13,22}$.

Los periodos sensibles son importantes durante el ciclo de vida, pues la exposición a un evento particular puede tener un efecto diferenciado dependiendo del momento de vida de una persona. Existen etapas de la vida en las que los efectos a la exposición de un suceso se magnifican, y ocasionan grandes cambios en las trayectorias vitales de quien los padece $e^{9,10,14}$. Por ejemplo, la adolescencia es una etapa sensible de exposición a distintos factores, y por ello es tan relevante en el ciclo vital de una persona: se experimentan cambios físicos y psicológicos, además del inicio de actividades que pueden cambiar la trayectoria vital, como el consumo de alcohol, drogas, el primer empleo o el inicio de la vida sexual $9,13,25,26$.

La epidemiología del curso de vida examina una variedad de procesos potenciales a través de los cuales las exposiciones que actúan en diferentes etapas de la vida pueden, individualmente o en combinación, influir en la morbilidad y los efectos a largo plazo ${ }^{1,14}$. Esta perspectiva epidemiológica ha conjuntado el enfoque del curso de vida y los estudios de salud, situando como principio de análisis los procesos potenciales de exposición que actúan a lo largo del ciclo vital y la manera en la que estos influyen en la prevalencia e incidencia de enfermedades ${ }^{11}$. La conjunción de los factores de exposición, el estrato socioeconómico, las distintas etapas del ciclo vital y los cambios epigenéticos de la población pueden analizarse desde la perspectiva del curso de vida y su influencia en la salud de la población.

Desde la perspectiva de la salud, se ha desarrollado una amplia gama de estudios con el enfoque del curso de vida, pero su influencia no ha sido suficiente en la investigación pediátrica ${ }^{13}$. Los estudios que se han realizado en pediatría son básicamente de seguimiento en la primera infancia y los periodos de vulnerabilidad $d^{9,20,21}$. El problema que se desea plantear es cómo utilizar el enfoque del curso de vida en la investigación pediátrica, para diseñar estudios que analicen el complejo proceso de la salud en la infancia, dado que una enfermedad grave en este periodo afectará de forma significativa el ciclo vital de una persona. Una transición tan importante como una enfermedad grave es un punto de inflexión que puede afectar definitivamente la trayectoria de vida de una persona, así como de la familia.

La propuesta de incluir el enfoque del curso de vida en la investigación pediátrica abarca una serie de aspectos: la teoría, el diseño, la recolección de datos y el análisis de información? 
El diseño que se propone para utilizar el enfoque del curso de vida en la investigación pediátrica es de corte longitudinal, pues se busca analizar los cambios que suceden en los sujetos de estudio a través del tiempo para establecer inferencias válidas sobre los fenómenos analizados y determinar los factores que están en juego para su explicación o la corrección a través de políticas públicas. Se deben realizar, cuando menos, dos seguimientos temporales; la amplitud de los intervalos de seguimiento dependerá de la manera en la que se manifieste el fenómeno que se está analizando'.

La elección del instrumento de medición supone una de las fases más importantes dentro del proceso de diseño de la investigación. Este se puede elegir por tres vías: un instrumento que ya se haya aplicado para el problema de estudio o para uno similar y que ya se encuentre validado; construir un instrumento propio y validarlo para el problema particular que se está estudiando; validar una escala que los investigadores responsables consideren «adecuada" para el constructo teórico que la respalda en la población particular que se desea estudiar ${ }^{27}$. En cualquiera de las tres opciones, se debe elegir un instrumento que pueda responder la pregunta de investigación y dar cuenta del problema que generó o impulsó la investigación.

Otro aspecto adicional del diseño es la forma de recabar la información: a partir de encuestas o entrevistas en profundidad. La elección de cada fuente de información dependerá del tamaño de la muestra, el tipo de estudio que se desarrolla -en el caso de una enfermedad poco frecuente, el tamaño de la muestra tiende a ser pequeño y sesgado-, los recursos financieros con los que se cuenta, el personal y disponibilidad del sujeto para participar y el tiempo de duración del estudio.

La recolección de la información es un aspecto fundamental, ya que existen distintas formas de captación dependiendo del estudio que se desarrolla y la frecuencia de la medición; por ejemplo, puede ser a través del trabajo de campo en los domicilios o unidades de salud, vía telefónica o a través de encuestas por Internet. De igual manera, la elección de una u otra forma de obtener la información depende de los recursos disponibles y del perfil profesional del personal que realizará el levantamiento, la localización geográfica, entre otros.

La fase final del diseño de un estudio de investigación es el análisis de los resultados, a partir de una metodología cuantitativa, cualitativa o mixta. La complejidad de la metodología seleccionada dependerá del tipo de instrumento seleccionado, de la información que se recabó, del grupo de analistas y del tiempo de seguimiento. La recomendación es recabar información cualitativa y cuantitativa; es decir, contar con distintas opciones de información para describir el fenómeno de estudio de forma más completa y desde distintos puntos de vista. Es importante señalar que los resultados de investigación a partir de este enfoque requerirán de algunos meses 0 años para ser publicados, debido al carácter longitudinal de esta perspectiva ${ }^{14,18,28}$. Con estos elementos conceptuales se puede iniciar un ejercicio de investigación que tenga como referencia conceptual el enfoque del curso de vida. Se presentan, a continuación, distintas investigaciones que han incorporado esta perspectiva en el diseño o en el marco teórico con el que sustentan el objetivo de estudio.

Una de las investigaciones que se ha diseñado a partir del enfoque del curso de vida es el Life-course Influences of Fetal Environments, un estudio de corte retrospectivo realizado en Detroit con mujeres afrodescendientes de 2009 a 2011. El estudio analiza cómo la movilidad social se encuentra asociada con dos resultados en el nacimiento: el ser pequeño para la edad gestacional o el parto prematuro ${ }^{29}$.

En 2005, el Contra Costa Health Services (CCHS) lanzó la llamada Life Course Initiative (LCI), con una periodicidad de 15 años, basada en la perspectiva del curso de vida. La LCl, implementada por el CCHS, está diseñada para reducir las desigualdades en los resultados del nacimiento, mejorar el potencial reproductivo y cambiar la salud de las generaciones futuras mediante la introducción de un enfoque longitudinal, integrado y ecológico para implementar programas de salud materna e infantil ${ }^{30}$.

El Estudio Nacional Longitudinal de Salud de los Adolescentes (The National Longitudinal Study of Adolescent Health), realizado por la Universidad de Carolina del Norte, se inserta como uno de los actores principales en el desarrollo de investigación del curso de vida por su vínculo innovador entre los factores socioeconómicos, de comportamiento y biológicos en una perspectiva de múltiples niveles ${ }^{28}$. Otra investigación que retoma esta perspectiva es el estudio HAPPIEE, desarrollado en países de la ex Unión Soviética sobre enfermedades cardiovasculares, el cual analiza en el largo plazo la influencia de fenómenos históricos en la salud de la población ${ }^{31}$.

Después de analizar algunos estudios que se han realizado desde la perspectiva de curso de vida, se exponen algunas fuentes de información que pueden utilizarse en la formalización de esta perspectiva. El Estudio Nacional de Salud y Envejecimiento en México 
elaboró una encuesta representativa a nivel nacional para la población de 50 años y más, que analiza las condiciones de envejecimiento y salud de la población en México. La encuesta que se generó es de carácter longitudinal, por lo que puede ser aprovechable por distintas concepciones teóricas y metodológicas, entre las que destaca el enfoque del curso de vida ${ }^{16}$.

En México, en 1998, se generó la primera encuesta biográfica, representativa para todo el país, denominada Encuesta Demográfica Retrospectiva, realizada para tres cohortes de edad (1936-1938, 1951-1953 y 1966-1968) en hombres y mujeres, las cuales aportan información sobre el ámbito familiar, laboral y migrato$r^{10}{ }^{16}$. Esta fuente de información no tiene una concepción metodológica fundamentada en esta perspectiva, pero sí aprovecha algunos elementos conceptuales y de diseño de la información.

Otra de las fuentes de información más importantes que se pueden analizar a partir del enfoque del curso de vida es la Encuesta Nacional sobre Niveles de Vida de los Hogares (ENNViH), de tipo longitudinal, multitemática y representativa de la población mexicana a nivel nacional, urbano, rural y regional. Actualmente, la ENNViH cuenta con tres rondas de levantamientos (2002, 2005-2006 y 2009-2012), en las que se han visto los cambios de la población desde la línea de base hasta 10 años posteriores a su inicio ${ }^{32}$.

El enfoque del curso de vida tiene múltiples aristas relacionadas con los temas de salud. Además de la creciente disponibilidad de fuentes de información y de procesamiento de datos, se han dado grandes avances en su formalización y uso. Por ejemplo, esta perspectiva puede nutrirse de la llamada biodemografía, con algunas mediciones antropométricas y el análisis de biomarcadores, para vincular el análisis de cambios sociales, individuales y a nivel biológico.

En el siguiente apartado se realizan las consideraciones finales y se ponen a discusión algunos aportes recientes al enfoque multidisciplinario de curso de vida, así como algunas de sus limitaciones.

\section{Consideraciones finales}

El enfoque del curso de vida representa una de las conceptualizaciones más integrales para el desarrollo de investigaciones en el campo social y de salud; sus postulados y principios pueden dar cuenta de procesos complejos, como el desarrollo de las vidas a lo largo de tiempo, los cambios y los efectos que estos cambios tuvieron en la configuración de las vidas individuales y del conjunto de la familia. Esta perspectiva puede servir para el análisis de procesos complejos o de larga duración, de cambios demográficos o de patrones de morbilidad-mortalidad, e incluso de patrones de cambio en el comportamiento social, como los patrones alimenticios y de consumo.

El curso de vida es una herramienta compleja, con múltiples aspectos a considerar en el diseño e implementación de investigaciones desde esta perspectiva. Las conclusiones pueden conducir a la elaboración de políticas públicas basadas en evidencia sólida cualitativa y cuantitativa, aunque con un elevado costo y un tiempo de espera prolongado en la obtención de resultados, ya que, por el tiempo de seguimiento, puede pasar un largo periodo para observar los fenómenos, por lo que los resultados de las cohortes pasadas pueden no ser tan aplicables a las generaciones actuales ${ }^{14}$. La información que se puede considerar relevante no se recopila al momento, sino de forma posterior, por lo que puede tomar décadas el alcanzar los grupos de edad con alta morbilidad y alta mortalidad $^{22,28}$.

Por lo anterior, no se ha desarrollado profusamente la investigación del enfoque del curso de vida en pediatría. Se da prioridad a la implementación y diseño de políticas públicas en adultos, debido a que los efectos en la salud de esta población pueden ser más convenientes que en los niños para muchos políticos, ya que los adultos pueden votar y las mejoras en su salud se traducen en productividad económica ${ }^{18,28}$.

Algunos de los resultados que resaltan del enfoque del curso de vida, llevados al extremo, pueden derivar en consideraciones e interpretaciones indebidas. Se debe proceder cautelosamente, ya que se puede invocar a los "genes" o a la "cultura» para explicar las disparidades raciales o étnicas en la salud, sin considerar los roles potenciales de las diferencias no medidas en experiencias sociales o económicas y en experiencias crónicas de estrés relacionadas con los bajos ingresos o la discriminación racial ${ }^{18}$.

Por último, existe la necesidad de traducir los nuevos conocimientos que se han integrado al enfoque del curso de vida en intervenciones y recomendaciones de políticas diseñadas para mejorar la salud de las personas, los grupos sociales y las sociedades a largo pla$z^{1}$. Los avances en la recolección, captura, análisis y procesamiento de datos pueden servir para utilizar fuentes de información más complejas. Es importante señalar que no solo es necesario recabar más información, sino vincular esta perspectiva con datos biomédicos para avanzar en la triple asociación: social, individual y biológica. 


\section{Conflicto de intereses}

Los autores declaran no tener ningún conflicto de intereses.

\section{Financiamiento}

El presente trabajo ha sido financiado por el Consejo Nacional de Ciencia y Tecnología (CONACyT) por medio del proyecto HIM 2016-029, titulado «Evolución económica y dinámica familiar en hogares con pacientes pediátricos a partir del diagnóstico de leucemia y durante el tratamiento", de la cuenta de inversión financiera CONACyT salud-2016-CO1-273470.

\section{Bibliografía}

1. Kuh D, Ben-Shlomo Y, Lynch J, Hallqvist J, Power C. Life course epidemiology. J Epidemiol Community Health. 2003:57:778-83.

2. Hareven TK, Masaoka K. Turning points and transitions: perceptions of the life course. J Fam Hist. 1988;13:271-89.

3. Hogan D, Mochizuki. Demographic transitions and the life course: lessons from Japanese and American comparisons. J Fam Hist. 1988; 13:291-305.

4. Elder G. The life course as developmental theory. Child Dev. 1998;69:1-12.

5. Hutchison E. A Life Course Perspective. En: Dimensions of human behavior: the changing life course. Thousand Oaks: SAGE Publications; 2005. p. 1-38.

6. Elder G. Time, human agency, and social change: perspectives on the life course. Soc Psychol Quarterly. 1994;57:4-15.

7. Sampson N, Parker E, Cheezum R, Lewis T, O'Toole A, Patton J, et al. A life course perspective on stress and health among caregivers of children with asthma in Detroit. Fam Community Health. 2013;36:51-62.

8. Kuruvilla S, Sadana R, Villar-Montesinos E, Beard J, Franz-Vasdek J, Araujo de Carvalho I, et al. A life-course approach to health: synergy with sustainable development goals. Bull World Health Organ. 2018;96:42-50.

9. Mullan Harris K. An integrative approach to health. Demography. 2010; 47:1-22.

10. Halfon N. Life course health development: a new approach for addressing upstream determinants of health and spending [Internet]. Washington: National Institute for Health Care Management; 2009. Disponible en: https://www.nihcm.org/pdf/ExpertVoices_Halfon_FINAL.pdf.

11. International Longevity Centre, World Health Organization. The implications for training of embracing. A Life Course Approach to Health [Internet]. Geneva: World Health Organization; 2000. Disponible en: https:// apps.who.int/iris/bitstream/handle/10665/69400/WHO_NMH_HPS_00.2_ eng.pdf? sequence=1\&isAllowed=y.
12. Fine A, Kotelchuck M. United States Maternal and Child Health Bureau. Rethinking $\mathrm{MCH}$ : the life course model as an organizing framework: concept paper. U.S. Department of Health and Human Services Health Resources and Services Administration Maternal and Child Health Bureau; 2010.

13. Fine A, Hampton P. Promoting Children's Long-Ter Health and Functioning: Applying a Life Course Approach to Pediatric Care for Children with Special Health Care Needs. Palo Alto: Lucile Packard Foundation for Children's Health; 2015.

14. Lynch J, Davey-Smith G. A life course approach to chronic disease epidemiology. Annu Rev Public Health. 2005;26:1-35.

15. Alwin D. Integrating varieties of life course concepts. J Gerontol B Psychol Sci Soc Sci. 2012;67B:206-20.

16. Blanco M. El enfoque del curso de vida: orígenes y desarrollo. Rev Latinoam Poblac. 2011:5:5-31.

17. Billari F. The life course is coming of age. Adv Life Course Res. 2009; $14: 83-6$.

18. Braveman P, Barclay C. Health disparities beginning in childhood: a life-course perspective. Pediatrics. 2009;124:S163-75.

19. Blanco M, Pacheco E. Trabajo y familia desde el enfoque del curso de vida: dos subcohortes de mujeres mexicanas. Pap Poblac. 2003:9:159-93.

20. Fine A, Kotelchuck M, Adess N, Pies C. Policy brief. A new agenda for $\mathrm{MCH}$ policy and programs: integrating a life course perspective. California: Contra Costa Health Services; 2009.

21. Shonkoff JP. Building a new biodevelopmental framework to guide the future of early childhood policy. Child Dev. 2010;81:357-67.

22. Blane D, Netuveli G, Stone J. The development of life course epidemiology. Rev Epidemiol Sante Publique. 2017;55:31-8.

23. Prus S. A life-course perspective on the relationship between socio-economic status and health: testing the divergence hypothesis. Can J Aging. 2004;23:145-53.

24. Aboderin I, Kalache A, Ben-Shlomo Y, Lynch JW, Yajnik C, Kuh D, et al. Life course perspectives on coronary heart disease, stroke and diabetes: key issues and implications for policy and research. Geneva: World Health Organization; 2002.

25. Institute of Medicine (U.S.). Breast cancer and the environment: a life course approach. Washington, DC: The National Academies Press; 2012.

26. Ragan D, Beaver K. Chronic offenders: a life-course analysis of marijuana users. Youth Soc. 2010;42:174-98

27. Anthoine E, Moret L, Regnault A, Sbille, Hardouin JB. Sample size used to validate a scale: a review of publications on newly-developed patient reported outcomes measures. Health Qual Life Outcomes. 2014:12:2.

28. Kim J, Durden E. Socioeconomic status and health over the life-course: an aging-vector approach [Pendiente de publicación]. University of Texas. Disponible en: https://paa2005.princeton.edu/papers/51531.

29. Osypuk T, Slaughter-Acey J, Kehm R, Misra D. Life-course social mobility and reduced risk of adverse birth outcomes. Am J Prev Med. 2016; 51:975-82.

30. Pies $C$, Parthasarathy $P$, Posner $S$. Integrating the life course perspective into a local maternal and child health program. Matern Child Health J. 2012;16:649-55.

31. Nilsson P. Public health and hypertension in Easter Europe-a life course perspective. J Hypertens. 2016;34:255.

32. Universidad Iberoamericana (UIA) y del Centro de Investigación y Docencia Económicas (CIDE). Encuesta Nacional sobre Niveles de Vida de los Hogares (ENNViH); 2019. Disponible en: http://www.ennvih-mxfls.org/ index.html. 